\title{
FURTHER OBSERVATIONS ON CORRECTING BEHAVIOUR IN BOXELDER BUGS
}

\author{
BY HUGH DINGLE \\ Department of Zoology, University of Michigan, Ann Arbor*
}

A previous paper (Dingle, 1961) described a characteristic behaviour pattern in boxelder bugs, Leptocoris trivittatus (Say) (Hemiptera, Coreidae), which was termed "correcting behaviour". When a bug was placed on a causeway with a $90^{\circ}$ forced turn, it would, if then given a choice, make a second opposite turn also of $90^{\circ}$. This second opposite turn was made in preference to proceeding straight ahead or to making a $90^{\circ}$ turn to the same side as the forced turn. In no case did a bug turn again to that same side (Dingle, unpublished observations). On a causeway which did not include a forced turn, the bugs went straight ahead at the choice point; they did not show a tendency to alternate during unrestricted movement. Two parameters which affected the frequency of occurrence of the opposite turn were the distances from the start to the forced turn and from the forced turn to the choice point. Increasing the former distance increased the correcting frequency while increasing the latter distance reduced it. The correcting behaviour was independent of running time for any section of the causeway, and of the total running time. There was no intentional reinforcement of any running pattern, and learning or conditioning were not involved.

The phenomenon of alternating turns appears to be a widespread one among animals in general, especially insects. It was found in 4 bugs and 2 beetles all from different families (Dingle, 1962), in mealworms (Grosslight \& Harrison, 1961; Akre, 1962), in cockroaches (Hullo, 1948), and in ants (Schneirla, 1929, but see Dingle, 1962). In other phyla it has been reported in forms as diverse as Paramecium (Lepley \& Rice, 1952) and rats (e.g. Witkin \& Schneirla, 1937). It does not occur in Planaria (Rice \& Lawless, 1957) and in at least one beetle (Dingle, 1962). Akre (1962) demonstrated alternation of turns on vertical as well as horizontal causeways in the red milkweed beetle, Tetraopes tetraophthalmus.

The present paper confirms and considerably extends my previous results with boxelder bugs.

*Present address: Mental Health Research Institute, University of Michigan, Ann Arbor, Michigan.
The relationships between start to forced turn distance, time of running, and correcting, are examined in more detail, and further features of correcting such as the effect of varying the forced turn are introduced. Previous experiments using a causeway with a choice of two alternative pathways only (Fig. 1A) revealed the existence of the behaviour when it was present to a marked degree, but did not give sensitive measurements of it. Accordingly some of the experiments reported here utilized causeways on which the bugs embarked upon a platform instead of reaching a fork. On the platform were arcs drawn at various distances from the choice point; the angles at which bugs crossed these arcs were then recorded. This method represented a refinement over the previous system where behaviour could be recorded only as a plus or a minus. Finally, in an attempt to identify the relevant sensory inputs, studies on blinded bugs are reported.

\section{Materials and Methods}

I collected most of the boxelder bugs used in these experiments in September and October when they regularly swarm on sunlit walls in the vincinity of female boxelder trees. They were kept until used in jars in a cool room (about 12 to $15^{\circ} \mathrm{C}$.) and fed on boxelder leaves as long as these were available and thereafter on sugar water. In this way they could be maintained until late February by which time the last of the animals collected in the autumn had died. Spring emergences usually occur in April at the sites of the fall swarming, and I collected more animals then. These latter, however, lived only a few weeks.

The causeways were constructed of wood or plasticine. They were placed in pans of water to prevent the bugs from stepping off; the wooden causeways were waterproofed and fastened down with strips of tape at the ends. The walking surfaces were 6.5 millimetres wide and were levelled by adjusting them to be just above the water surface over their entire length; all junctions were smooth. In those experiments involving a causeway with a choice of two alternatives 
(Fig. 1A) the two final routes were orientated so that they were at equal angles to parallel rays of light coming from a window (Dingle, 1961). When the bugs debouched on a platform,

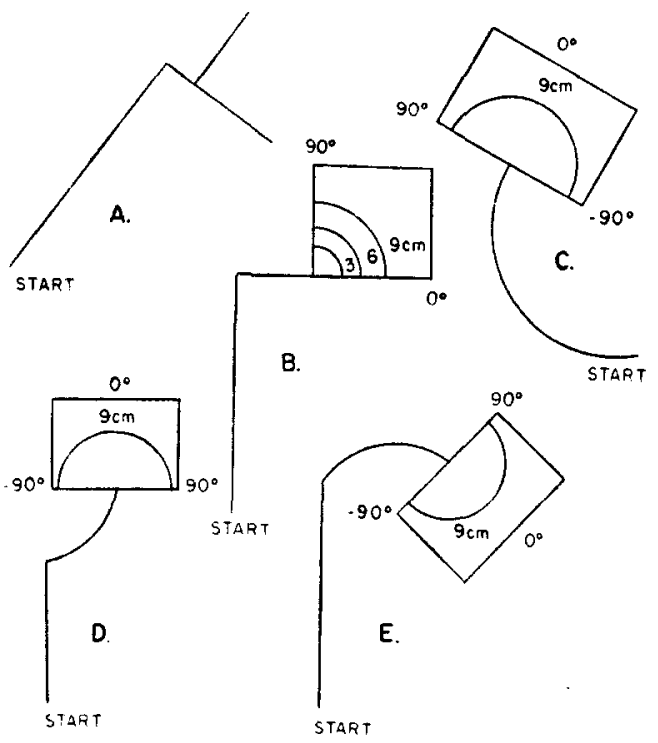

Fig. 1. Causeways used for the various experiments (explanations in text).

this was directly under a single light bulb with the rest of the room darkened. The various causeways are shown in Fig. 1.

All non-parametric statistical tests used are discussed in Siegel (1956); where no reference is given for these tests, that is the source. The design of each of the experiments is given with it in the appropriate section of the Results.
Results

Effect of Varying the Distance from Start to Forced Turn

In experiments previously reported (Dingle, 1961), I showed that as the distance from start to forced turn was increased, the frequency of turning as against going straight at a subsequent two-alternative choice point also increased. To obtain a more direct measure of the turning effect, I used a causeway which offered the bugs a platform at the choice point instead of the two alternatives offered in the earlier trials (Fig. 1B). Arcs were drawn on the platform at 3,6, and 9 centimetres from the choice point and were marked at $10^{\circ}$ intervals. As a bug crossed one of the 3 arcs, the degree interval at which it crossed was recorded; three successive arccrossings at $90^{\circ}$ indicated a full turn on the platform and three at $0^{\circ}$ indicated that the bug proceeded straight ahead with no turn. At each of 4 distances from start to forced turn, 5,10 , 15 and $20 \mathrm{~cm}$., I ran 50 bugs over the causeway once each; at $0 \mathrm{~cm}$., i.e. making no forced turn, and at $12.5 \mathrm{~cm}$. I ran 25 bugs. The distance from forced turn to choice point was always $5 \mathrm{~cm}$. These experiments were done in a room lit only by a 100 watt bulb placed $25 \mathrm{~cm}$. directly above the centre of the platform. The complete results and the $p$ values of the differences between them are shown in Table I; the data from the $9 \mathrm{~cm}$. arc are graphed in Fig. 2A. It is obvious that the angle at which the bugs crossed the platform underwent a marked change between the 10 and $12.5 \mathrm{~cm}$. distances from start to forced turn. Comparisons of the data for the two distances from all 3 arcs yielded p's $<.01$ (Table I) using the low power efficiency median

Table I. Bugs Run on a Platformed Causeway. Data from causeway shown in Figure 1A. Average angles (in degrees) at which bugs crossed ares after travelling indicated distance from start to forced turn. Each reading an average of 50 bugs run once apiece except those at $12.5 \mathrm{~cm}$. and $0 \mathrm{~cm}$. which are for 25 bugs. The 3 readings at a given distance are for the $50(25)$ bugs.

\begin{tabular}{|c|c|c|c|}
\hline $\begin{array}{l}\text { Start to Forced } \\
\text { Turn distance }\end{array}$ & $3 \mathrm{~cm}$ & $\begin{array}{l}\text { Ares } \\
6 \mathrm{~cm}\end{array}$ & $9 \mathrm{~cm}$. \\
\hline $20 \mathrm{~cm}$ & $47 \cdot 0^{\circ}$ & $51 \cdot 1$ & $54 \cdot 7^{\circ}$ \\
\hline $15 \mathrm{~cm}$ & $46 \cdot 2^{\circ}$ & $51 \cdot 6$ & $54.5^{\circ}$ \\
\hline $12.5 \mathrm{~cm}$ & $43 \cdot 6^{\circ}$ & $47 \cdot 4$ & $50 \cdot 4^{\circ}$ \\
\hline $10 \mathrm{~cm}$. & $20 \cdot 9^{\circ}$ & $24 \cdot 9$ & $26 \cdot 6^{\circ}$ \\
\hline $5 \mathrm{~cm}$ & $13 \cdot 7^{\circ}$ & $18 \cdot 6$ & $21.5^{\circ}$ \\
\hline $0 \mathrm{~cm}$ & $16 \cdot 6^{\circ}$ & $17 \cdot 4$ & $18 \cdot 0^{\circ}$ \\
\hline
\end{tabular}




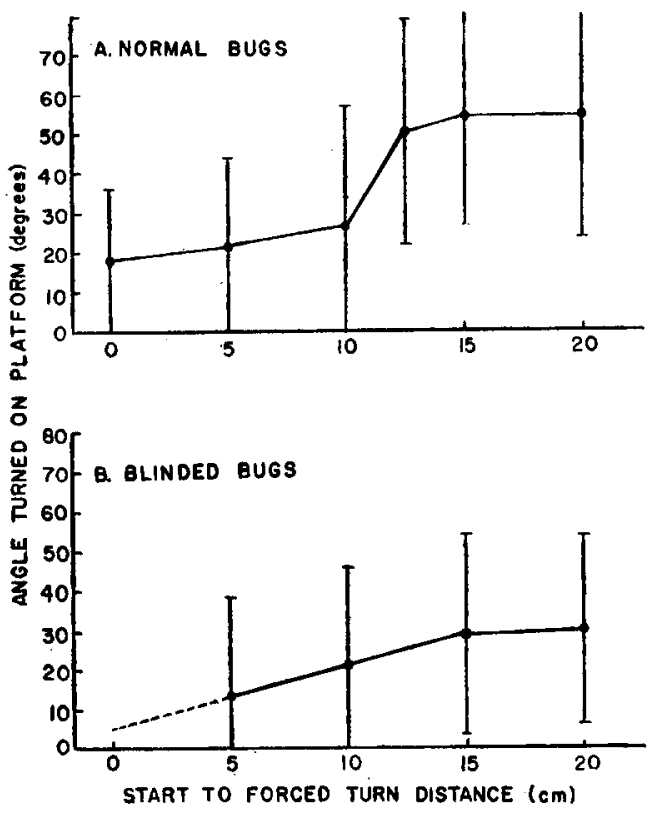

Fig. 2. Graphs of $9 \mathrm{~cm}$. arc data from Tables $I$ and V. Vertical lines are + or -1 standard deviation.

test. The data for the 15 and $20 \mathrm{~cm}$. (p's $>.50$ in all cases). For 5 and $10 \mathrm{~cm}$. data there is a significant difference at the .05 level for the $3 \mathrm{~cm}$. arc, and for the other 2 arcs the low $p$ values are certainly suggestive of a difference. The graph in Fig. 2 thus seems to show that the angle turned on the platform increased sigmoidally as distance from start to forced turn increased. The standard deviations are, however, unfortunately large, the possibility of a single direct rise rather than a sigmoidal increase cannot be eliminated. The previous data on distance from start to forced turn, from a causeway with a choice point of two alternatives, also indicated a sigmoidal or sudden increase in the percentage of turns at the choice point (Dingle, 1961).

The angles at which the bugs crossed the 3 arcs show that they did not simply turn at the choice point and then proceed in a straight line, but continued to turn after embarking on to the platform. Using the data from all 250 bugs over all trials for the 3 arcs, I found $\cdot 10>\mathrm{p}>\cdot 05$ using the parametric $\mathrm{F}$ test (Fisher \& Yates, 1948). While these values are not significant at the $\cdot 05$ level, they do at least indicate differences in the angles at which bugs crossed the respective arcs.

The platformed causeway was also used for an experiment on the effect of delay time on the angle turned at the choice point. After it had travelled $15 \mathrm{~cm}$. from start to forced turn, each bug was covered with a small wooden box. Some movement was possible beneath the box and a few bugs were able to turn completely around or upside down; most, however, were in the same position when released as when first covered. The bugs were kept beneath the box for various periods of time and then released to proceed the remaining $5 \mathrm{~cm}$. to the forced turn and thence to the platform. The angle at which each bug crossed the $9 \mathrm{~cm}$. arc on the platform was recorded, and the lighting was again a 100 watt bulb directly above the centre of the platform.

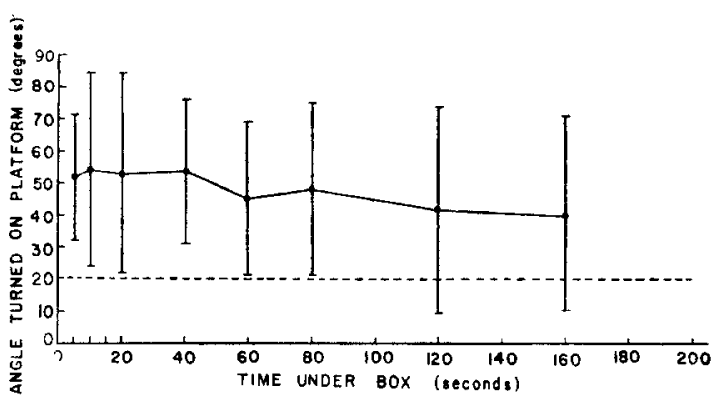

Fig. 3. The effect of delays on the angle turned on the platform. Each point an average from 25 bugs. Dotted line gives lower asymptote (see text). Vertical lines are - + or - 1 standard deviation.

The results are given in Fig. 3 with each point representing an average from 25 bugs. The lower asymptote (dotted line) is taken from the $5 \mathrm{~cm}$. data in Table I and represents the average angle, approximately $20^{\circ}$, at which the bugs would have been expected to cross the $9 \mathrm{~cm}$. arc had they run only $5 \mathrm{~cm}$. start to forced turn in the first place (the equivalent of an infinite delay under the box after running the first $15 \mathrm{~cm}$.). As can be seen, delays of up to 40 seconds did not affect the bugs' mean turning angle; it was essentially the same as when they had travelled the $20 \mathrm{~cm}$. from start to forced turn unhindered (see Table I). Following this there was an apparent slow decline in the angle of crossing with each longer delay, but even with a delay of 160 seconds the angle turned, $41^{\circ}$, was still conspicuously above the theoretical lower asymptote. The effect of the distance from start to forced turn was thus a persistent one.

\section{Effect of Varying the Angle of the Forced Turn}

For this experiment I used causeways each with a choice point with two alternatives (Fig. 
1A; the platformed causeway had not at the time of these trials been introduced into the experiments), and arranged the forced turns so that the bugs would move through angles ranging from $90^{\circ}$ to $0^{\circ}$ (no turn). For all the causeways the distance from start to forced turn was $15.0 \mathrm{~cm}$. and from forced turn to choice point, $7.5 \mathrm{~cm}$. A total of 100 bugs was run on each causeway, with each bug run but once; the results are shown in Table II. These indicate a change in corrections between $60^{\circ}$ and $75^{\circ}$ of turn, with a greater number at $75^{\circ}$. The difference between the results for these two angles

Table II. Effect of Forced Turn Angle on Correcting with Forced Turn to Choice Point and Start to Forced Turn Distances Held Constant.

\begin{tabular}{|c|c|c|}
\hline $\begin{array}{c}\text { Angle of } \\
\text { forced turn }\end{array}$ & \multicolumn{2}{|c|}{ Number of Bugs } \\
\hline $90^{\circ}$ & Correcting & Not correcting \\
\hline $75^{\circ}$ & 47 & 53 \\
60 & 44 & 56 \\
$45^{\circ}$ & 23 & 77 \\
$30^{\circ}$ & 19 & 81 \\
$0^{\circ}$ & 22 & 78 \\
\hline
\end{tabular}

is significant (using $x^{2}, \mathrm{p}<\cdot 01$ ), while that between any other two angles is not. Rather than a steady increment in correcting as the amount of turn increased, there was thus a certain critical angle at which a marked and significant increase occurred. The relatively low overall frequency of corrections was a consequence of the longer distance from forced turn to choice point (see Dingle, 1961).

\section{Curved Causeways}

In order to vary the nature of the forced turn, I ran bugs on curved causeways, i.e. on continuous forced turns. These causeways, constructed of plasticine, were arcs of circles of different radii; at the end of the causeway the bugs embarked on to a platform where the direction in which they turned was recorded. The arrangement is shown in Fig. 1C. The bugs travelled for either 12 or $24 \mathrm{~cm}$. on causeway arcs having radii of 5,10 , and $15 \mathrm{~cm}$. On the platform, a semicircle of radius $9 \mathrm{~cm}$. was drawn with its centre at the "choice point" junction with the curved causeway; this semi-circle was marked off at $10^{\circ}$ intervals from $+90^{\circ}$ to $-90^{\circ}$. The causeway curved to the right so that a rightangled turn to the left on the platform meant crossing the $9 \mathrm{~cm}$. semicircle at $+90^{\circ}$, going straight meant crossing at $0^{\circ}$, and turning to the right at right angles meant crossing at $-90^{\circ}$. Twenty-five bugs were run over each distance on each curved causeway each bug being run only once. Again the only light was a 100 watt bulb $25 \mathrm{~cm}$. directly above the centre of the platform. The results are shown in Table IIIA. Table IIIB shows the degrees of arc traversed by the bugs in the different situations.

Table IIIA. Bugs on a Curved Causeway.

Causeway shown in Fig. 1C. Readings show mean angle (in degrees) at which 25 bugs crossed a $9 \mathrm{~cm}$. arc on a platform after travelling 12 or $24 \mathrm{~cm}$. on arcs of given radii.

\begin{tabular}{|c|cc|}
\hline $\begin{array}{c}\text { Radius of } \\
\text { circling arc }\end{array}$ & Distance & travelled \\
\hline Normal bugs circling to right & $12 \mathrm{~cm}$. & $24 \mathrm{~cm}$. \\
\hline $5 \mathrm{~cm}$. & $+19^{\circ}$ & $+42 \cdot 2^{\circ}$ \\
$10 \mathrm{~cm}$. & $+10 \cdot 4^{\circ}$ & $+28 \cdot 6^{\circ}$ \\
$15 \mathrm{~cm}$. & $+3 \cdot 4^{\circ}$ & $+11 \cdot 4^{\circ}$ \\
\hline Normal & bugs circling to left \\
\hline $5 \mathrm{~cm}$. & $-25^{\circ}$ & $-43^{\circ}$ \\
\hline Blinded & bugs circling to right \\
\hline $5 \mathrm{~cm}$. & $+16.4^{\circ}$ & $25 \cdot 6^{\circ}$ \\
\hline
\end{tabular}

Table IIIB. Degrees of Arc Traversed by Bugs when Travelling 12 or $24 \mathrm{~cm}$. on Ares of Given Radii.

\begin{tabular}{|c|c|c|}
\hline \multirow{2}{*}{$\begin{array}{l}\text { Radius of } \\
\text { circling arc }\end{array}$} & \multicolumn{2}{|c|}{ Distance travelled } \\
\hline & $12 \mathrm{~cm}$ & $24 \mathrm{~cm}$. \\
\hline $5 \mathrm{~cm}$ & $136^{\circ}$ & $272^{\circ}$ \\
\hline $10 \mathrm{~cm}$ & 68 & $136^{\circ}$ \\
\hline $15 \mathrm{~cm}$ & $45^{\circ}$ & $90^{\circ}$ \\
\hline
\end{tabular}

The data reveal that a bug forced to curve to the right tended to turn left when it came to the platform $(a+$ turn $)$, just as it did when it had been forced to make an abrupt turn to the right, above. Furthermore, the smaller the radius of curvature, and the greater the distance walked along a curve of given radius, the more pronounced was the subsequent opposite turn 
on the platform. The mean angle turned there by the 25 bugs running $24 \mathrm{~cm}$. on the arc of $5 \mathrm{~cm}$. radius was greater than that of the 25 bugs running the same distance on the $10 \mathrm{~cm}$. arc $(\mathrm{p}<\cdot 04$ with a one-tailed Mann-Whitney $U$ test); and the mean angle after the arc of $10 \mathrm{~cm}$. radius was greater than that after the arc of $15 \mathrm{~cm}$. radius ( $\mathrm{p}<\cdot 01$ with a two-tailed test). The results of $24 \mathrm{~cm}$. runs on arcs of all three radii differed significantly from those of $12 \mathrm{~cm}$. runs ( $\mathrm{p}<\cdot 01$, two-tailed).

Table IIIA also shows the results of one of the two control series used for the experiments with curved causeways. The bugs were made to circle to the left instead of to the right on an arc of radius $5 \mathrm{~cm}$.; in this case they made a right turn upon arrival at the platform, as predicted. In the second control series the bugs were run on an entirely straight $24 \mathrm{~cm}$. causeway. The mean angle turned by 25 bugs on the platform was $+4.2^{\circ}$ which, as determined by the parametric $t$ test (Fisher \& Yates, 1948), was not significantly different from the expected value of $0^{\circ}(\mathrm{p}<\cdot 20)$.

While proceeding along the curved causeway, most bugs ran fairly smoothly down the centre of the runway. Some, however, seemed to make a series of small abrupt turns, at times starting off the edge of the runway and turning back on to it when their front tarsi touched the water surface. But the behaviour of these bugs on the platform did not differ on the average from that of animals which ran smoothly. There was no tendency to turn along the edge of the platform. This is consistent with behaviour noted previously (Dingle, 1961) and by Akre (1962).

To examine more directly the relationship between a curved causeway and an abruptly angled one, I performed two further experiments. The first experiment utilized a causeway with a straight section followed by a curved section ending on a platform (Fig. 1E). The straight section of the causeway was $12 \mathrm{~cm}$. long and led directly into an arc of $5 \mathrm{~cm}$. radius and $12 \mathrm{~cm}$. long $\left(136^{\circ}\right)$. The platform had a semicircle drawn $9 \mathrm{~cm}$. from its junction with the curved causeway and marked off in $10^{\circ}$ intervals from $+90^{\circ}$ to $-90^{\circ}$. Again the light was a 100 watt bulb $25 \mathrm{~cm}$. over the platform. Ten bugs were run 5 times on the curved section only and on the entire causeway. The trials were randomized. For the curved section only the average turn in all trials was $+38^{\circ}$ while for the entire causeway it was $+63^{\circ}(\mathrm{p}<.05$ for a two-tailed Wilcoxon test). Because of differences in ex- perimental conditions (for example, these tests were performed in daylight) the results are not comparable with those using curved causeways alone (Table IIIA), but they do clearly confirm that a forced turn is corrected whether it be abrupt or continuous. They also confirm that a prior straight run strengthens the effect of a forced turn.

In the second experiment the causeway had a $20 \mathrm{~cm}$. straight run and then an abrupt $90^{\circ}$ turn into an arc $8 \mathrm{~cm}$. long $\left(90^{\circ}\right)$ of $5 \mathrm{~cm}$. radius ending on a platform (Fig. 1D). Twenty-five bugs which ran only the curved part of the causeway, circling to the left in this case, turned through a mean angle of $+25 \cdot 2^{\circ}$ on the platform, a right turn. The 25 bugs which began their run at the start of the straight causeway and thus made a sharp $90^{\circ}$ turn before circling left through a total of another $90^{\circ}$, crossed the platform semicircle at a mean angle of $+3 \cdot 8^{\circ}$. This was not significantly different from the expected value of $0^{\circ}(\mathrm{p}<-20$ for the $t$ test $)$. The two means of $+3 \cdot 8^{\circ}$ and $+25 \cdot 2^{\circ}$ were significantly different ( $\mathrm{p}<.03$ for a one-tailed Mann-Whitney $U$ test). The $90^{\circ}$ curving turn to the left thus exactly counteracted the effect of the previous sharp $90^{\circ}$ turn to the right as far as the subsequent free path on the platform was concerned.

\section{Blinded Bugs}

Bugs were blinded by covering the eyes and ocelli with several coats of quick drying paint and were run in dim light. Three causeways, the right-angled one with a choice point offering only two alternatives (Fig. 1A), the right-angled one ending on a platform (Fig. 1B), and the curved one ending on a platform (Fig. 1C), were used with blinded bugs.

The causeway with a choice of two alternatives was $15 \mathrm{~cm}$. long from start to forced turn and $3.75 \mathrm{~cm}$. from forced turn to choice point. The results for 10 bugs are shown in Table IV. These differed significantly from those obtained previously (Dingle, 1961) with normal bugs on a causeway with two similar alternatives but no forced turn at all $(\mathrm{p}<.02 \mathrm{Kolmogorov-Smirnov}$ two-sample test). Thus, blinding reduced the tendency to make a correcting turn at the choice point, but not to the insignificant level obtained in the absence of any forced turn.

The results for blinded bugs run over the platformed causeway (Fig. 1B) are given in Table $\mathrm{V}$ and should be compared with those for normal bugs in Table 1 . The data for the $9 \mathrm{~cm}$. arc are graphed in Fig. 2B. These blinded 
Table IV. Blinded, Partially Blinded and Normal Bugs.

Data for blinded, partially blinded, and normal bugs on a causeway with a $90^{\circ}$ forced turn and a choice point of 2 alternatives and for normal bugs on a causeway with no forced turn grouped in classes according to number of corrections made. Data for normal bugs from Dingle (1961).

\begin{tabular}{|c|c|c|c|c|c|c|c|c|c|c|c|c|}
\hline & \multicolumn{12}{|c|}{ Number of corrections } \\
\hline & 0 & 1 & 2 & 3 & 4 & 5 & 6 & 7 & 8 & 9 & 10 & $\mathbf{N}$ \\
\hline Number of Blinded bugs & 0 & 0 & 1 & 2 & 1 & 3 & 2 & 1 & 0 & 0 & 0 & 10 \\
\hline $\begin{array}{l}\text { Number of bugs lower halves of } \\
\text { eyes blinded }\end{array}$ & 1 & 0 & 1 & 2 & 1 & 1 & 2 & 0 & 2 & 0 & 0 & 10 \\
\hline $\begin{array}{l}\text { Number of bugs upper halves of } \\
\text { eyes blinded }\end{array}$ & $\mathbf{0}$ & 0 & 0 & 0 & 1 & 1 & 0 & 2 & 2 & 1 & 3 & 10 \\
\hline Number of normal bugs & 0 & 0 & 2 & 0 & 2 & 3 & 1 & 4 & 9 & 4 & 5 & 30 \\
\hline Number of normal bugs no forced turn & 7 & 2 & 1 & 0 & 0 & 1 & 0 & 0 & 0 & 0 & 0 & 11 \\
\hline
\end{tabular}

Table V. Blinded Bugs on a Platformed Causeway. Data as in Table I.

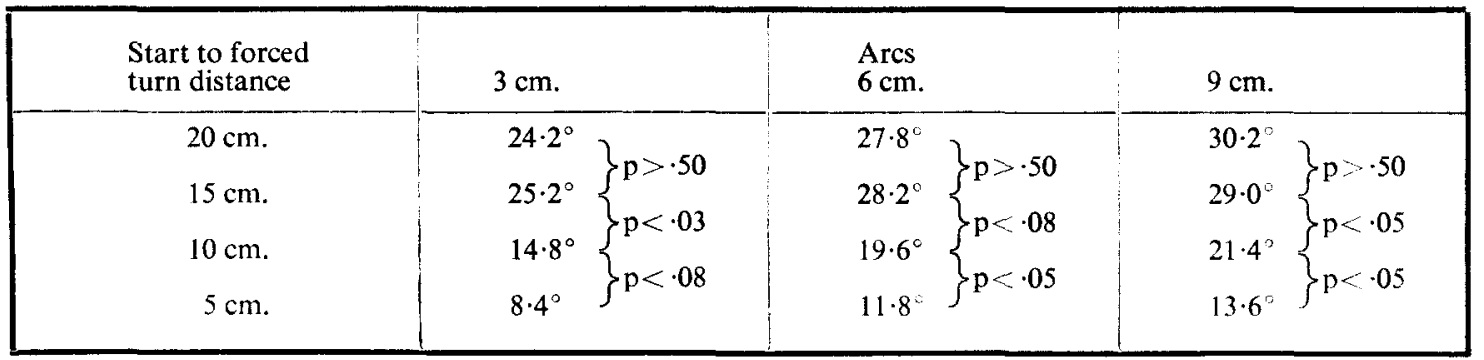

animals, unlike the normal ones, did not show a marked increase in angle turned on the platform when the distance from start to forced turn was increased from 10 to $15 \mathrm{~cm}$. Rather, the angle turned appears to have increased by equal increments as that distance was increased from 5 to 10 and from 10 to $15 \mathrm{~cm}$., and, as in the normal bugs, there was no further change between 15 and $20 \mathrm{~cm}$.; the p values using a onetailed Mann-Whitney $U$ test are given in the table. The data for the $9 \mathrm{~cm}$. arc from the 15 $\mathrm{cm}$. runs of normal and blinded animals gave quite different means ( $\mathrm{p}<\cdot 01$; two-tailed MannWhitney $U$ test). The $10 \mathrm{~cm}$. straight run appears to have had the same effect on the blinded animals as the $5 \mathrm{~cm}$. straight run had on the normal animals, while the $15 \mathrm{~cm}$. straight run seems to have had a somewhat larger effect on the blinded than the $10 \mathrm{~cm}$. run had on the normal animals. Note also that in blinded bugs, as in the normal, the correcting turn appeared to be a continuous one as revealed by the mean angles at which the three successive arcs were crossed.
Blinded bugs were also run for 12 and 24 $\mathrm{cm}$. along the curved causeway of $5 \mathrm{~cm}$. radius (Fig. 1C). After the $12 \mathrm{~cm}$. run the average angle turned was $+16.4^{\circ}$, and after $24 \mathrm{~cm}$. it was $+25 \cdot 6^{\circ}$. As mentioned above, normal bugs running straight for $24 \mathrm{~cm}$. made a mean turn of $+4.2^{\circ}$ upon arrival at the platform; the mean angle turned by the blinded bugs after running $24 \mathrm{~cm}$. on the curved causeway was significantly different ( $p<\cdot 01$, two tailed Mann-Whitney $U$ test). The bugs were circling to the right, and a positive angle means that they made left turns upon arriving at the platform; this behaviour is similar to that noted with normal bugs. Again as with normal bugs, the left turn was more pronounced the longer the run on the curved causeway had been, although the differences in mean angle between 12 and $24 \mathrm{~cm}$. here were not significant ( $\mathrm{p}>\cdot 11$, two-tailed Mann-Whitney $\mathrm{U}$ test). As in the previous experiment, the mean angle turned by the blinded bugs was less than that of normal animals. The turn of normal bugs after a $24 \mathrm{~cm}$. run on a curve of radius $5 \mathrm{~cm}$. was significantly greater than the turn 
of the blinded bugs under the same conditions ( $\mathrm{p}<\cdot 05$, one-tailed Mann-Whitney $U$ test).

The mean time taken to travel from start to forced turn in the 10 runs of the 10 blinded bugs on the causeway with a final choice of two alternatives (Fig. 1A), did not differ significantly from 10 normal bugs chosen at random from among those run on the same causeway $(\mathrm{p}>\cdot 10$; two-tailed Mann-Whitney U test).

\section{Partial Blinding}

To delimit the part of the eye necessary for normal correcting behaviour, I painted over the upper and lower halves, respectively, of the eyes of two groups of 10 bugs. The ocelli were covered when the upper halves were painted. These animals were then run on the causeway with a choice of two alternatives. The results are given in Table IV along with those from fully blinded bugs; they show clearly that bugs with the lower halves of the eyes blinded behaved like the fully blinded bugs, while those with only the upper halves blinded behaved in the normal manner previously reported (Dingle, 1961). Sight in the lower half of the eye thus appeared necessary for behaviour equivalent to that of a normal animal in these experiments with the causeway lying on the bottom of a white pan.

\section{Discussion}

"Centrifugal swing" (Schneirla, 1929) in which an animal would be "thrown" by its "momentum" against the outside of a forced turn and hence would be likely to make an opposite turn at a choice point has been invoked at various times to explain alternating turns. To the grounds already given for rejecting this hypothesis for boxelder bugs (Dingle, 1961), there may now be added the results of the experiment in which a box was put over the animal prior to the forced turn (Fig. 3). When the box was removed, the bugs started from a standstill and proceeded $5 \mathrm{~cm}$. to the forced turn. The run prior to the delay under the box obviously contributed to the subsequent correcting; on an hypothesis of "centrifugal swing" it should not have done so. Centrifugal swing has also been rejected for mealworms (Tenebrio) by Grosslight \& Harrison (1961) who largely eliminated it in their mazes, but found that alternation of turns still occurred.

Grosslight \& Harrison (see also Grosslight \& Ticknor, 1953) and Lepley \& Rice (1952) propose instead the concept of "reactive inhibition" to account for behaviour similar to correcting in mealworms and Paramecium respectively. The term goes back to Hull (1943) who defined it as a negative motivational tendency, which declines with time, not to repeat an action; a right turn, for instance, would reduce the probability of an immediately succeeding right turn. Both Lepley \& Rice and Grosslight \& Harrison point out a decline in "correcting" as distance after the forced turn increases. They then indicate that this decline with distance represents a decline with time, thus equating time and distance. A decline of reactive inhibition with time follows as a logical deduction from Hull's theory (Dember \& Fowler, 1958).

The concept of "reactive inhibition" is inappropriate for correcting behaviour in boxelder bugs for three reasons: (1) The action not to be repeated in this case would be a turn in the direction previously forced; accordingly, the distance from start to forced turn, i.e. activity which preceded the turn, should not affect correcting; and it does (Table I). (2) If reactive inhibition decays with time, then a longer time between forced turn and choice point should result in a decrease in correcting; previous results (Dingle, 1961) have shown that given the same distance from forced turn to choice point, correcting was independent of the time taken to travel that distance or, in other words, time and distance cannot be equated. (3) In the experiments on Paramecium and mealworms the animals were forced to make a choice at a $\mathrm{T}$ junction where one alternative was a turn to the same side as the forced turn. In the experiments with the bugs, the alternatives were an opposite turn or going straight, with no possibility of repeating the previous turn; and the bugs made the opposite turn. The conclusion from the foregoing is not that the bugs were failing to repeat a previous action but that they were performing specifically the opposite action.

The basis for this opposite action seems to be in the interaction of events occurring during the run from start to forced turn and at the forced turn. Fig. 2 indicates that an excitatory state is built up between the start and forced turn in normal bugs. Whatever the nature of the excitatory state, it endures for at least 40 seconds (Fig. 3). Even after considerably longer delays the mean turning angle was only slightly affected; at 160 seconds, for example, it was reduced from about $50^{\circ}$ to $41^{\circ}$.

Presumably events at the forced turn interact with the established excitatory state to produce 
the opposite turn at the choice point. Table II indicates that with the distance from start to forced turn and from forced turn to choice point held constant, a certain critical amount of forced turning, between $60^{\circ}$ and $75^{\circ}$, is necessary if the opposite turn (correcting) is to follow. The relevant factors are at present unknown; perhaps a significant change in the walking pattern or pattern of visual inputs is involved. Experiments are currently in progress to investigate this problem further (see also discussion below).

One might at first expect a similar change in behaviour when a critical amount of turning had occurred on curved causeways. The data in Table III, however, reveal no such threshold effect. Two possible reasons for this suggest themselves: (1) at a forced turn there is a sudden change in direction imposed on a bug in a short period of time while on a curved causeway there is a gradual change over a relatively long period; and (2) the effect of distance travelled before experiencing a forced turn may be different from the effect of distance travelled while turning on a curved causeway.

The data in Table III reveal that there are also quantitative differences in behaviour between bugs on curved causeways (Fig. 1C) and those run on straight causeways with an abrupt turn (Fig. 1B). For instance, bugs travelling straight for $20 \mathrm{~cm}$., then being forced to make an abrupt $90^{\circ}$ turn, and then proceeding $5 \mathrm{~cm}$. to a platform, turned at a mean angle of $54.7^{\circ}$ (at the 9 $\mathrm{cm}$. arc) on the platform (Table I). Bugs which travelled as far $(24 \mathrm{~cm}$.) and were likewise forced to make a $90^{\circ}$ turn, but this time circling on a curve of $15 \mathrm{~cm}$. radius, subsequently turned at a mean angle of only $11.4^{\circ}$ on the platform (Table IIIA). In considering only the curved causeway data, one observes that bugs travelling $24 \mathrm{~cm}$. on an arc of $10 \mathrm{~cm}$. radius, or $12 \mathrm{~cm}$. on an arc of $5 \mathrm{~cm}$. radius, had in both cases turned through $136^{\circ}$ altogether (Table IIIB), yet in the former case the mean angle subsequently turned on the platform was $28 \cdot 6^{\circ}$ while in the latter it was $19^{\circ}$ (Table IIIA). There thus seem to be two major factors which determine the extent of a turn on a platform at the end of a causeway. The first is the distance travelled, and the second is the rate of change of direction while travelling. The two parameters can influence each other. The further effect of total amount of forced turn is contingent on these two factors and exerts independent in- fluence only when they are held constant (see above).

As far as relevant sensory inputs are concerned, there can be little doubt that vision is important in correcting behaviour. Correcting was reduced by about a half in blinded bugs on a causeway with a choice of two alternatives (Table IV). On the platformed causeway the correcting turn was again reduced, and the shape of the curve of effect of distance from icart to forced turn was also changed (Fig. 2). On the platformed causeway, blinded bugs required a greater distance than normal ones to show the same amount of correcting turn, and they never reached the maximum amount of turn shown by normal bugs. Similarly, blinded bugs running $24 \mathrm{~cm}$. on a curved causeway of $5 \mathrm{~cm}$. radius showed a decrease after a $12 \mathrm{~cm}$. run. The data in Table IV indicate that the important visual input was received through the lower halves of the eyes in the given conditions.

Of other sense organs, the antennae do not seem to play a part since their removal did not reduce the frequency of the behaviour (Dingle, 1961). Interestingly, however, removal of the antennae in the red milkweed beetle Tetraopes tetraophthalmus did result in a reduction in frequency (Akre, 1962).

Obviously, then, vision is important to correcting behaviour, but equally obvious and equally important it is not the only factor involved. Although reduced, the behaviour is still manifest when vision is absent. Whatever is functioning in that absence, and proprioception would seem the most likely possibility, must be coequal in importance with vision. The nature and operation of the relevant factors, however, remain to be investigated.

\section{Summary}

1. After making a forced turn on a causeway, boxelder bugs make a turn on a platform in a direction opposite to the forced turn. The angle turned on the platform increases abruptly or in sigmoid fashion as the start to forced turn distance increases. Blinded bugs show a similar effect, but the angle is reduced and the increase is not sigmoid or abrupt.

2. After running for most of the distance between start and forced turn, bugs can be delayed for 40 seconds or more without effect on mean angle turned on the platform.

3. At a certain critical forced turn angle, between $60^{\circ}$ and $75^{\circ}$, the frequency of turns at a choice point offering either a turn opposite to 
the forced turn or a straight path markedly increases (with the start to forced turn and forced turn to choice point distances kept constant).

4. If bugs were made to circle on a causeway, they would turn opposite to the direction of circling upon arrival at a platform. The amount of turn increased as distance run increased or as the radius of the circling arc decreased. Two important factors thus seem to be distance travelled and rate of change of direction.

5. Blinding reduces the degree of opposite turning made at a choice point after making an abrupt forced turn and the opposite direction turned on a platform after traversing a circling arc. Opposite turning is, however, still present to some extent. It is the lower halves of the eyes which are important.

6. Explanations of the above behaviour based on "centrifugal swing" and "reactive inhibition" are rejected.

7. Events occurring at the forced turn probably interact with an excitatory state built up between the start and the forced turn to cause opposite turning at a choice point. Whatever the excitation state, it builds up in sigmoid or abrupt fashion and endures for a period of at least 40 seconds.

8. Vision certainly, and proprioception probably, are important factors in the performance of the above behaviour.

\section{Acknowledgments}

This paper contains portions of a thesis submitted as part of the requirements for a Ph.D. at the University of Michigan. I should like to thank my doctoral committee, Drs. L. B. Slobodkin, R. D. Alexander, Morris Foster, and R. W. Gerard who offered a great deal of advice and encouragement. Dr. F. E. Smith gave helpful advice on statistical methods. The manuscript was read by Drs. J. S. Kennedy, who also made many helpful suggestions regarding the experiments, and G. M. Hughes; their comments and criticisms have been very valuable. My wife, Geraldine Dingle, and Mrs. G. A. Fuller gave needed and appreciated help in typing the manuscript.

Portions of this research were carried out during the tenure of a National Science Foundation Co-operative Graduate Fellowship.

\section{R E F ER E N C E S}

Akre, R. D. (1962). Correcting behavior by insects on vertical and horizontal mazes. Master's Thesis, Kansas State University.

Dember, W. N. \& Fowler, H. (1958). Spontaneous alternation behavior. Psychol. Bull., 55, 412-428.

Dingle, H. (1961). Correcting behavior in boxelder bugs. Ecology, 42, 207-211.

Dingle, H. (1962). The occurrence of correcting behavior in various insects. Ecology, 43, 727-728.

Fisher, R. A. \& Yates, F. (1948). Statistical Tables for Biological, Agricultural and Medical Research. London: Oliver \& Boyd.

Grosslight, J. H. \& Harrison, P. C. (1961). Variability of response in a determined turning sequence in the mealworm (Tenebrio molitor): an experimental test of alternative hypotheses. Anim. Behav., 9, 100-103.

Grosslight, J. H. \& Ticknor, W. (1953). Variability and reactive inhibition in the meal worm as a function of determined turning sequence. $J$. comp. physiol. Psychol., 46, 35-38.

Hull, C. L. (1943). Principles of Behavior. New York: Appleton-Century-Crofts, Inc.

Hullo, A. (1948). Role des tendances motrices et des données sensorielles dans l'apprentissage du labyrinthe par les blattes (Blatella germanica). Behaviour, 1, 297-310.

Lepley, W. M. \& Rice, G. E. (1952). Behavior variability in paramecia as a function of guided act sequences. J. comp. physiol. Psychol., 45, 283-286.

Rice, G. E. \& Lawless, R. H. (1957). Behavior variability and reactive inhibition in the maze behavior of Planaria drotocephala. J. comp. physiol. Psychol., 50, 105-108.

Schneirla, T. C. (1929). Learning and orientation in ants. Comp. psychol. Monogr., 6, 1-142.

Siegel, S. (1956). Nonparametric Statistics for the Behavioral Sciences. New York: McGraw-Hill.

Witkin, H. A. \& Schneirla, T. C. (1937). Initial maze behavior as a function of maze design. J. comp. Psychol., 23, 275-304.

(Accepted for publication 12th June, 1963; Ms. number: 348). 